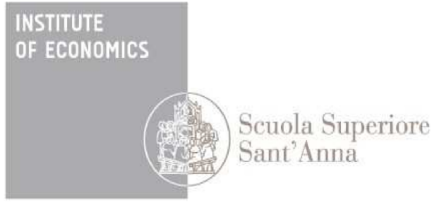

LEM | Laboratory of Economics and Management

Institute of Economics

Scuola Superiore Sant'Anna

Piazza Martiri della Libertà, 33 - 56127 Pisa, Italy ph. +3905088.33 .43$

institute.economics@sssup.it

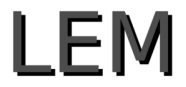

Working Paper Series

\title{
The International Trade Network in Space and
} Time

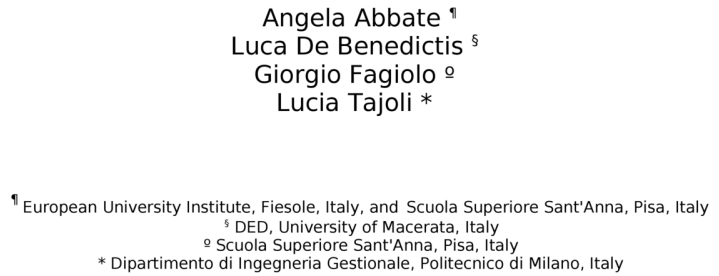

Angela Abbate"

Luca De Benedictis ${ }^{\S}$

Giorgio Fagiolo 은

Lucia Tajoli*

"European University Institute, Fiesole, Italy, and Scuola Superiore Sant'Anna, Pisa, Italy

${ }_{5}^{5}$ DED, University of Macerata, Italy

@ Scuola Superiore Sant'Anna, Pisa, Italy

* Dipartimento di Ingegneria Gestionale, Politecnico di Milano, Italy 


\title{
The International Trade Network in Space and Time*
}

\author{
Angela Abbate ${ }^{\dagger} \quad$ Luca De Benedictis ${ }^{\ddagger} \quad$ Giorgio Fagiolo ${ }^{\S}$ \\ Lucia Tajoli $₫$
}

October 2012

\begin{abstract}
This paper studies how the structure of the International Trade Network (ITN) changes in geographical space and along time. We employ geographical distance between countries in the world to filter the links in the ITN, building a sequence of sub-networks, each one featuring trade links occurring at similar distance. We then test if the topological properties of ITN subnetworks change as distance increases. We find that distance strongly impacts, in non-linear ways, the topology of the ITN. We show that the ITN is disassortative at long distances while it is assortative at short ones. Similarly, the main determinant of the overall high ITN clustering level are triangular trade triples between geographically close countries. This means that trade partnership choices are differentiated over different distance ranges. Such evidence robustly arises over time and after one controls for the economic size and income of trading partners.
\end{abstract}

Keywords: International Trade, Network Analysis, Distance.

JEL Classification: C02, F10, F14.

* The authors thank the participants to the ARS'11 Conference in Naples (June 2011), the NAIT workshop in Macerata (February 2012) and the ETSG Conference in Leuven (September 2012) for their insightful comments. The financial support of the PRIN2009 tradenetworks.it project is graciously acknowledged.

${ }^{\dagger}$ Department of Economics, European University Institute, Via della Piazzuola 43, Florence 50133, Italy and Scuola Superiore Sant'Anna, Piazza Martiri della Liberta' 33, Pisa 56127, Italy. a. abbate@sssup.it

${ }^{\dagger}$ DED - University of Macerata - Via Crescimbeni 20, Macerata 62100, Italy. +390733258236. debene@unimc.it

${ }^{\circledR}$ Scuola Superiore Sant'Anna, Piazza Martiri della Liberta' 33, Pisa 56127, Italy. giorgio.fagiolo@sssup.it

"Dipartimento di Ingegneria Gestionale, Politecnico di Milano - piazza Leonardo da Vinci 32, Milano 20133, Italy. +390223992752. lucia.tajoli@polimi.it. 


\section{Introduction}

After a relatively long period of neglect, geography and distance have re-gained momentum in international trade analysis. Both in models based on monopolistic competitive homogeneous firms a là Krugman (1980), and in models displaying heterogeneous productivities, as in Eaton and Kortum (2002) and Melitz (2003), distance plays a fundamental role in generating a wedge between domestic and international prices. This wedge is generally attributed to international trade costs (Anderson and van Wincoop, 2004). Also in the empirical analysis of trade flows, the overwhelming use of the gravity equation in the prediction of the volume of bilateral trade flows is emphasizing once again the persistent role of distance in international exchanges.1 The economic relevance of space, i.e. the cost associated to physical distance, has also influenced macroeconomists in singling it out as a fundamental explanation of discrepancies between canonical models and empirical evidence (Obstfeld and Rogoff, 2001).

The inclusion of distance in international trade models and empirics has certainly shortened the gap between international trade and economic geography. The inclusion of concepts such as market potential (Head and Mayer, 2010), remoteness (Wei, 1996; Coe et al., 2007) or multilateral resistance (Anderson and van Wincoop, 2003) in international trade analysis encouraged researchers to move from the two-country-world of international trade theory and the bilateral focus of trade empirics, to a more global analysis, where the possible role of third countries - or more generally of the structure of the links among all countries - in influencing country dyadic relations is taken into account ${ }^{2}$

Nevertheless, especially in applied analyses, the concept of distance is still essentially considered as an absolute, bilateral concept (e.g., as an individual characteristic of each single country $i$ with respect to any other country $j$ ). Indeed, even the widespread use of more relative concepts such as multilateral resistance in gravity estimation boils down, in practice, to employing country fixed-effects in panel data Hummels, 1999 ; Feenstra, 2002; Redding and Venables, 2004), i.e. labeling the cost of distance with a bilateral attribution instead of a multilateral one. The consequence is that the deep, structural dimension of multilateral resistance is still largely under-explored.

In recent years, however, a network approach to international trade has started to

\footnotetext{
${ }^{1}$ In the gravity model of international trade, popularized by Tinbergen (1962), the aggregate value of trade between a pair of countries is proportional to the product of their incomes and inversely related to the distance between them. In the the traditional log-log version of the gravity equation, the estimated coefficients correspond to elasticities, and distance enters (log) linearly in the estimate of bilateral trade flows. See Disdier and Head (2008) for a meta-analysis of the empirical literature on the gravity model, Santos Silva and Tenreyro (2006) for the possible implication of the inclusion of distance in a log form, and De Benedictis and Taglioni (2011) and Anderson (2011) for recent overviews of the gravity model.

${ }^{2}$ The literature on "third country effects" is still very sparse. See Baltagi et al. (2007) for an application to foreign direct investments, and Egger and Larch (2008) and Chen and Joshi (2010) for an application to regional trade agreements. In a different perspective, some authors have looked at the role of "third countries" in the transmission of shocks, see Abeysinghe and Forbes (2005) and Dees and Saint-Guilhem (2011).
} 
uncover the properties of the web of trade flows by focusing precisely on the structural, multilateral side of international trade relations (Fagiolo et al., 2009; De Benedictis and Tajoli, 2011). This approach begins by describing international-trade flows using graphtheoretic structures where countries (nodes) are linked by arcs or edges representing (possibly weighted) import-export relationships between countries. This techniques allow one to get rid of purely dyadic relations among countries and enhance the importance of network topology and the roles played by country relative positions in the network. This literature hints to the existence of interesting statistical regularities in the topology of trade networks, e.g. the disassortative nature of the network (i.e. the fact that highlyconnected countries tend to be linked to countries which are not well-connected) or the relatively high level of clustering (i.e. a high likelihood that any two trade partners of a country are themselves trade partners). Furthermore, it has been shown that the structural properties of the trade network may have important consequences as far as shock transmission and country growth are concerned (Kali and Reyes, 2007; Kali et al., 2007).

Traditionally, network analyses of international-trade flows did not explicitly take on board geography. In other words, the network of trade flows has never been embedded in a geographical space. Therefore, no attempts have been made in linking topological (geodesic) distances among countries to their geographical counterparts $3^{3}$ In this paper, we begin to bridge this gap by including geographical space in a network analysis of international-trade flows. To do so, we employ geographical distances between countries to filter the ITN and build sub-networks of countries located at similar geographical distances. We then test if the topological properties of these subnetworks are robust to geographical distance. The short answer we get is: no. We find that the effect of distance on trade networks is strongly non-linear. Many of the properties that one typically observes at the aggregate level (i.e. without considering distance) are not robust to a geographical breakdown. For example, the network is disassortative at long distances, while it is assortative at short ones, the switch happening at a distance of approximately $4000 \mathrm{~km}$. A similar finding applies to clustering: short-distance country triplets are the major contributors to the strong level of overall clustering. By repeating this exercise over time, we show that this evidence is persistent from year 1970 to year 2000, and that it remains consistent even after one explicitly takes into account the size of countries involved in trade. This brings an important insights for the analysis of trade networks and international trade in general: the structure of international trade flows is not isomorphic along distance.

The rest of the paper is organized as follows. In section 2 we review the literature

\footnotetext{
${ }^{3}$ This is also the case for network analysis in general. There are however notable exceptions in the area of geography, urban systems and transport analysis. See Barthelemy (2011) for a review of the issue, and Wilson (2000) and Haggett and Chorley (1969).
} 
dealing with geographical distance and trade models. Section 3 discusses the main findings of network-related literature on trade. In section 4 we describe the data used in our analysis and the methodology used. Section 5 presents our main results. Finally, section 6. concludes.

\section{International trade and distance}

Distance matters in international trade. This is so whenever the movements of goods and services across space implies some costs. Econometric estimates of the constant elasticity of trade to geographical distance provide a measure of the relevance of those costs and their persistence over time (Brun et al., 2005), ranging within an interval of -0.7 and -1.2 (Disdier and Head, 2008). This means that on average, all else equal, countries twice apart show a bilateral volume of trade which is approximately half the one of neighboring countries.

In line with this evidence, the most recent trade models embody the idea that the geographical distance between the country where goods and services are produced and the country where they are sold and consumed has a negative effect on trade flows between the two countries. There may be several reasons why this is the case. Taking an extreme view, on the one hand, trade costs can operate proportionately to distance, increasing variable costs or reducing the quantity of the exported goos that reaches the foreign market, as in Samuelson's iceberg trade-cost formulations. On the other hand, trade costs can follow a Bernoulli process associated to the acquisition of an export status by firms extending their activities from a purely domestic context to an international one. In this case, distance acts as a fixed sunk cost, that can be specific to the each foreign market or payed once-and-for-all when the firm, having payed the fixed sunk trade cost, has acquired the knowledge necessary to be active in any foreign market.4

The implications of such alternative views in terms of trade partnership and trade flows among countries are clearly not the same. If trade costs are directly proportional to distance, trade partnerships would expand radially around countries and trade volumes would smoothly decrease with distance, conditional on the size of the foreign market. Conversely, if trade costs are fixed entry costs, trade partnership would be a more discontinuous process, and the selection of partners and trade volumes would be somehow independent on the distance between the home and foreign market.

Trade economists have dealt with this issue in terms of extensive margin and intensive margin of trade (i.e. new trade links vs. increasing strength of pre-existing trade links) $5^{5}$

\footnotetext{
${ }^{4}$ See Redding (2011) for a review of the recent theoretical literature on heterogeneous firms and trade and on the role of trade costs in this stream of research. The fundamental paper by Anderson and van Wincoop (2004) gives a comprehensive overview of the issue.

5The dimensions through which aggregate trade is typically split in margins are many. Just to fix ideas, the extensive margin can be considered at the country level (new export markets or countries
} 
The consensual piece of evidence is that distance operates mainly through the extensive margin, as shown by Lawless (2010) and Bernard et al. (2007). Within a gravity-like set-up, using firm data on US exports, both papers decompose exports into the average export sales (the intensive margin) and the number of exporting firms (the extensive margin), while Bernard et al. (2007) also includes a second component to the extensive margin: the average number of products sold on foreign markets by exporting firms. In Lawless (2010) distance has a negative effect on both margins, but the magnitude is considerably larger for the extensive margin. In Bernard et al. (2007) both the number of exporting firms and the number of exported products are linearly decreasing in the distance to the destination country; in contrast, the average export value is increasing in distance ${ }^{6}$ The negative effect of distance strongly emerges only at the extensive margin.

The theoretical paper by Chaney (2008), along the lines of the literature on heterogeneous firms that focuses on the micro-foundation of the gravity equation (see also Anderson and van Wincoop (2003), Melitz (2003), Eaton and Kortum (2002), and Helpman et al. (2008)), reinforces the empirical evidence, showing that fixed costs affect only the extensive margin of trade. In facts, in Lawless (2010), the time-invariant variables that may influence bilateral trade, capturing the role played by fixed cost (i.e. language, internal orography, infrastructure and import barriers) work through the extensive margin.

This evidence, distinguishing the role of distance between its effect on the extensive and intensive margin, has an implicit echo in the different analyses of the internationaltrade network (ITN). The notions of extensive and intensive margins, at the country level, can be easily applied to network analysis. Generally speaking, at the country level the extensive margin of trade is the change in the number of trade partners of a country over time, either importers or exporters or both, which coincides with the change in the number of the links of a country in the trade network. Conversely, the intensive margin is associated with the change of of a country's pre-existing trade flows, e.g. the sum of the pre-existing weights of the links of a country.

The second issue that is worth discussing in the context of the gravity equation is the way distance is included as a covariate of bilateral trade flows. As we mentioned already, the cost of distance is in general interpreted as a fixed (sunk) cost or as a variable cost,

from which imports are coming from); at the sectoral level (new product lines get activated in export or import data, at the sectoral level); at the firm level (new firms enlarge their reference market beyond national boundaries); or at the product level (multi-product firms start selling new product varieties abroad).

6 Bernard et al. (2007) speculate on the potential explanation for this surprising result. If the cost of exporting depends on quantity or weight rather than on values, an increase in distance may lead to $a$ change in the composition of exports towards higher-value commodities, "... for which it is profitable to incur the fixed and variable trade costs of servicing the remote and small foreign market. The differences in value-to-weight ratio across commodities may in turn be explained by differences in their quality ... If the change in composition towards higher-value commodities is sufficiently large, the average value of exports per product per firm may be increasing in distance." 
with different implications. Even in its seminal work, Tinbergen (1962) distinguishes between variable costs (distance) and fixed costs, approximated by the cost-reducing effect of an adjacency/contiguity dummy, that takes the value 1 when two trading countries share a common border and 0 otherwise. Since McCallum (1995) it is evident that crossing the national border is costly, and doing it only once is advantageous. Doing it more than once, as for countries which are not adjacent, is more costly. If we add to that many other variables that since Tinbergen (1962) have been augmenting the gravity equation to account for fixed costs due to a different or similar historical trade pattern (being part of the same empire, being colonies or colonizers, being enemies or allies), signing trade agreements or sharing a common currency, using the same or similar languages, sharing the same formal or informal institutions (legal system, religions, property rights, reciprocal trust), or even the same genetic map, we end-up with the same logical structure, where the cost of distance is $(\log )$ linearly related to geography and discontinuously related to the degree of (broadly conceived) similarity of the countries involved in trade.

Focusing on the first component, in principle, given the different nature of trade costs, there is no reason to believe that distance should be related to trade in a (log)linear manner. As pointed out by Bernard et al. (2007) and Hummels (2007) among others, transportation costs can induce a selection among the goods that are sold in distant markets, and the average value of exports could increase in distance precisely to compensate the increase in trade costs. Even for a given distance, trade costs are much dependent on the characteristics of specific goods, such as fragility, perishability, size or weight. In aggregate terms, trade costs would depend on trade composition and would be country specific, affected by country's remoteness and sectoral specialization. Such non-linearities are usually addressed empirically using a log-log specification 7 This procedure can be however costly, de-facto removing all zero flows and generating a selection in the data. The role played by fixed cost is identified only on those flows that are able to cover those fixed cost and not on those that are not able to afford it.

In some cases, the assumption of a $(\log )$ linear cost of space is substituted with some ad hoc functions. In Eaton and Kortum (2002) the distance effect is associated to six nonoverlapping distance intervals: [0, 375); [375, 750); [750, 1500); [1500,3000); [3000, 6000); and [6000, maximum], measured in miles. Anderson and Yotov (2012) uses the same specification decomposing the distance effect into four different elasticities corresponding to the four non-overlapping distance intervals: [0, 3000); [3000, 7000); [7000, 10000); [10000, maximum], measured in kilometers. It is amazing that neither one of the two papers explains why this stepwise functional forms has been adopted. Hillberry and Hummels 2005) bring some illuminating evidence on why the Eaton and Kortum (2002)

\footnotetext{
${ }^{7}$ For an analysis of the implications of estimating the gravity model in a log-linear form with heteroskedastic errors see Santos Silva and Tenreyro (2006) and De Benedictis and Taglioni (2011) for a survey of non-linear estimators in the context of the gravity model.
} 
specification might be ad hoc but meaningful: there is an extraordinary difference between short and long-distance trade. Using highly disaggregated data at the spatial and sectoral level on manufacturers' shipments within the United States, they find that the pattern of shipments is strongly localized. Shipments within 5-digit zip codes, with a median radius of only 4 miles, are 3 times larger than shipments outside the zip code. The analysis shows that distance reduces aggregate trade values primarily by reducing the number of commodities shipped and the number of establishments shipping. " ... Extensive margins are particularly important over very short distances".

The analysis that will follow adds a complementary motive to the micro evidence put forward by Hillberry and Hummels (2005), and brings support to the distinction between short and long-distance trade, thus giving a further support to the Eaton and Kortum (2002) specification of the effect of distance on trade. More specifically, we embed a multilateral perspective to international trade, based on network theory, in an explicit geographic dimension, in order to ask whether the structure of international trade is isomorphic across distance.

\section{Complex Networks and International Trade}

In the last years, there was an increasing surge of interest in applying a complex-network approach to the study of international trade. 8

The International Trade Network (ITN), aka World-Trade Web (WTW) or World Trade Network (WTN), is defined as the graph of import/export relationships between countries in a given year $t$. The resulting graph, $\mathcal{G}_{t}=\left(\mathcal{V}_{t}, \mathcal{L}_{t}\right)$, where $n_{t}=\left|\mathcal{V}_{t}\right|$ is the number of countries constituting the vertices (or nodes) of the graph, and $m_{t}=\left|\mathcal{L}_{t}\right|$ is the number of existing directed trade links (or arcs), gives rise to network $\mathcal{N}_{t}=\left(\mathcal{V}_{t}, \mathcal{L}_{t}, \mathcal{P}_{t}, \mathcal{W}_{t}\right)$ - where $\mathcal{P}_{t}$ is the vertex value function including the exogenous or endogenous properties of vertices, and $\mathcal{W}_{t}$ is the line value function including the exogenous or endogenous weights of links - which could be a binary (unweighted) network, $\exists \mathcal{W}_{t}=\mathcal{A}_{t}$ where $\mathcal{A}_{t}$ is, for every year $t$, a $n \times n$ 0-1 matrix, if only the presence/absence of a positive trade flow is considered, or could be a weighted network, $\exists \mathcal{W}_{t} \neq \mathcal{A}_{t}$, if links have different intensities, according to e.g. the value of the bilateral trade flows.

A lot of effort has been recently put forward in uncovering the topological properties of the ITN architecture, both at the aggregate and at the product-specific level (see Fagiolo et al., 2010, Barigozzi et al., 2010, for a review of the main results). Understanding the topological properties of the ITN from a complex-network perspective Albert

${ }^{8}$ See for example Li et al. (2003); Serrano and Boguñá $(2003) ;$ Garlaschelli and Loffredo (2004,|2005); Reichardt and White (2007); Serrano et al. (2007); Bhattacharya et al. (2008, 2007); Garlaschelli et al. (2007); Tzekina et al. (2008); Fagiolo et al. (2008); Reyes et al. (2008); Fagiolo et al. (2009); De Benedictis and Tajoli (2011). 
and Barabási, 2002; Dorogovtsev and Mendes, 2003), and their evolution over time, is fundamentally important to study issues such as economic globalization, the spreading of international crises, and the transmission of economic shocks (Helliwell and Padmore, 1985; Artis et al., 2003; Forbes, 2002).

A network approach, by focusing on direct as well as indirect relationships between countries, is able to single out the role of each countries in the complex web of world trade interactions. This is a remarkable change with respect to traditional analyses. Indeed, the standard approach to international-trade empirics employs statistics that fully characterize the profile of a country in the system by referring mainly to its bilateraltrade direct linkages. Whereas direct bilateral trade linkages are known to be one of the most important channels of interaction between world countries (Krugman, 1995), recent studies show that they can only explain a small fraction of the impact that an economic shock originating in a given country can have on another one, which is not among its direct-trade partners (Abeysinghe and Forbes, 2005). Along similar lines, Squartini et al. (2011a b) show that knowledge of country-specific indicators such a the number of trade partners, total imports or total exports (which only take into account direct bilateral links in the ITN) is not enough in the weighted ITN to characterize higher-order moments of the distribution of trade relationships, involving for example the trade behavior of the partners of a given country, the likelihood that any two trade partners of a vertex are themselves partners, etc..

In order to fully account for system-wide phenomena such as globalization and crises diffusion, a more detailed knowledge of the local and global topological properties of the network is therefore required. This means, in other words, acquiring a better understanding of the presence and importance of trade paths connecting any pair of non-direct trade partners and, more generally, of topological indicators proxying the likelihood that economic shocks might be transmitted between any two countries (Kali and Reyes, 2007). This, in turn, has been shown to help explaining patterns of macroeconomic dynamics related to, e.g., growth and development (Kali et al., 2007; Reyes et al., 2008).

The issue of assortativity is central in empirical studies of real-world networks. More generally, one asks whether there exists any assortative mixing between nodes, i.e. similar nodes are linked or not. If one takes similarity as connectivity, assortative networks feature well-connected nodes joining to other well-connected nodes, whereas in disassortative networks strongly connected nodes are linked to weakly-connected ones. Newman (2002, 2003) have shown that nodes' connectivity in many social networks tends to be positively correlated. McPherson et al. (2001) cite over one hundred studies that have observed homophily in some form or another. Examples range from company director networks, co-authorship and collaboration networks, or the network of email address books. On the contrary, most biological networks (protein-protein interaction network in the yeast cell, metabolic networks in bacteria, food webs) or technological networks (the Internet 
at the Autonomous System level, the network of hyperlinks between pages in the World Wide Web, etc.) appear to be disassortative. Networks in economic contexts may have features of both technological and social relationships (Jackson, 2010).

In spite of the evidence produced in the above mentioned studies on the topological properties of the ITN, complex-network approaches to trade have generally neglected the issue of geographical distance and space (for a review on spatial networks, see Barthelemy, 2011). To begin bridging this gap, this paper explicitly takes on board geographical space in the way ITN graphs are defined. More precisely, we build trade graphs by filtering international trade flows so as to build trade-network structures where the presence of any bilateral link is conditioned on the geographical distance between its two end nodes (countries). We will therefore analyze the topological properties discussed above conditional on countries' distance.

\section{Data and Methodology}

In our analysis of the ITN, we employ the dataset made available by Subramanian and Wei (2007) $!^{9}$ This dataset includes values of aggregate bilateral imports in U.S. dollars, at current prices, as reported by the importing country in the IMF Direction of Trade Statistics, and then deflated by US CPI (at 1982-83 prices). We use data from 1970 to 2000, focusing on export flows only 10

More formally, let $n_{t}$ be the number of countries present in the database in year $t$, where a country $i$ is said to enter the database if there is at least a positive import or export flow associated to it. Define, as in section 3, $\mathcal{W}_{t}$ as the $n_{t} \times n_{t}$ weight matrix of the corresponding weighted directed ITN, where the generic element of $\mathcal{W}_{t}$, labeled as $w_{t}(i, j)$ represents the logarithmic transformation of positive-valued export flows from country $i$ to $j$ in year $t$ (and zero if the corresponding trade flow is zero) ${ }^{11}$ We also define the time- $t$ binary matrix $\mathcal{A}_{t}$ as the binary $n_{t} \times n_{t}$ matrix whose generic element $a_{t}(i, j)=1$ if and only if $w_{t}(i, j)>0$, and zero otherwise. Therefore, we constructed both a weighted directed and a binary directed representation of the ITN. The binary directed representation gives us information about the presence or absence of trade partnerships, whereas the weighted directed representation adds to the binary structure information about the heterogeneity of export flows carried by each link.

\footnotetext{
${ }^{9}$ The dataset used by Subramanian and Wei (2007) is downloadable from the website http://www. nber.org/ wei/data.html.

${ }^{10}$ The numerical results for imports are different in magnitude but they display very similar patterns. We don't report them here for ease of exposition, but the full set of analyses including both exports and imports is available upon request.

${ }^{11}$ We use a logarithmic scale instead of a linear one in order to make easier any comparison of our results with those from standard log-log gravity-equation formulation. To allow for meaningful comparisons across years we also re-scale link weights by logs of yearly total world exports so as to account for the overall increase in total trade over time.
} 
The properties of the binary directed representation of the ITN built with this dataset were examined by De Benedictis and Tajoli (2011) and Duenas and Fagiolo (2011). Table 1 presents some descriptive statistics.

Table 1: Summary statistics.

\begin{tabular}{lrrrr} 
& 1970 & 1980 & 1990 & 2000 \\
\hline Countries (No.) & 130 & 143 & 145 & 157 \\
Trade Flows (No.) & 6593 & 8180 & 10289 & 11938 \\
Density & 0.393 & 0.403 & 0.493 & 0.487 \\
\hline Countries making up to 50\% of trade & 7 & 9 & 7 & 11 \\
Flows making up to 50\% of trade & 71 & 88 & 67 & 77 \\
Countries making up to 90\% of trade & 65 & 75 & 77 & 78 \\
Flows making up to 90\% of trade & 793 & 893 & 747 & 854 \\
\hline Average trade flow & 50.72 & 57.20 & 70.96 & 76.04 \\
Median trade flow & 54.50 & 60.00 & 64.00 & 70.00 \\
\hline
\end{tabular}

Source: IMF, Directions of Trade Statistics.

Over time, the ITN displays an increasing number of participating countries. Entry of new countries in the database is due to the presence of at least a new positive trade flow involving the entrant, and may be possibly caused either by the availability of new data or by the actual entry of the country in the international trade market. New trade links, however, seem to increase more than quadratically with the number of participating countries, as shown by the rising density of the network over time ${ }^{12}$ Note also that the number of countries making up a high percentage (50\% or $90 \%$ respectively) of total trade tends to increase over the years, hinting to an increasing engagement in trade by a larger number of countries. However, given that also the total number of reported trading countries in our sample is also becoming larger, this figure does not necessarily imply a significant decline in the concentration of world trade.

The ITN as defined above does not consider the effect of geographical distance on trade. To properly account for such an effect, we begin considering the original binary $\left(\mathcal{A}_{t}\right)$ and weight $\left(\mathcal{W}_{t}\right)$ matrix (from now on we remove the time label to simplify the notation). To any given link $i j$ between countries $i$ and $j$ (i.e., to each ordered pair $(i, j)$ such that $a(i, j)=1)$, we associate the geographical distance $d(i, j)$, computed using the great-circle distance measure between the capital cities of the two countries. This allows us to build a geographical-distance matrix $\mathcal{D}=\{d(j, i)\}$, whose generic element is equal to the geographical distance between countries $i$ and $j$ whenever the correspondent trade link exists, and zero otherwise. Note that this matrix is by construction symmetric and possibly changes through time.

\footnotetext{
${ }^{12}$ The density in a directed network is computed as the ratio between existing links and the maximum number of possible links, i.e. $\gamma_{t}=\frac{m_{t}}{m_{\max }}=\frac{m_{t}}{n_{t}\left(n_{t}-1\right)}$. For example, in 1970 one gets: $\gamma_{1970}=\frac{6593}{130 \times 129}=$ 0.393
} 
Figure 1 plots a kernel-density estimation for the distribution of the logs of geographical distances between world countries in year 2000 (i.e. of the positive upper-diagonal entries of the matrix $\mathcal{D}$ ). It is easy to see that the distribution of the logs of distance is skewed to the left and presents a peak at large distances.

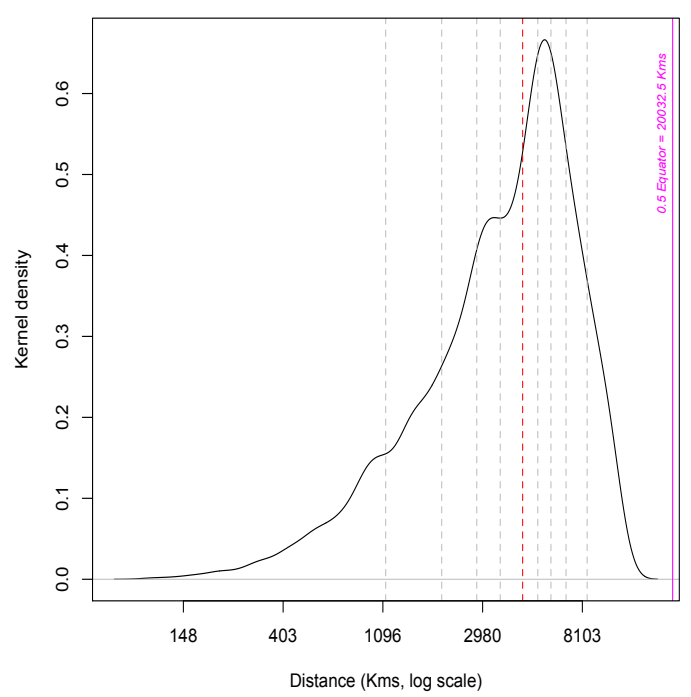

Figure 1: The distribution of geographical distance between country pairs. Kernel density estimate of the distribution of distance (logarithmic scale). The red vertical line indicates the median value of the distribution, the gray lines indicate the deciles of the distribution, while the violet vertical line, bounding the distribution to the right, indicates half of the Equator distance.

Table 2 breaks down the values of total trade (in percent) according to the different deciles of the distribution of distance among countries. The table suggests that most international trade occurs at relatively short (less than $2000 \mathrm{~km}$ ) and intermediate distances, but the distribution is very uneven.

Table 2: Share of total trade (in percentage) of each distance decile, by decade

\begin{tabular}{lcccccccccc}
\hline $\begin{array}{l}\text { Decile } \\
\text { Interval } \\
(\mathrm{km})\end{array}$ & $\begin{array}{c}1 \text { st } \\
\text { 0-1128 }\end{array}$ & $\begin{array}{c}\text { 2nd } \\
1129-1978\end{array}$ & $\begin{array}{c}\text { 3rd } \\
\text { 1979-2811 }\end{array}$ & $\begin{array}{c}4 \text { th } \\
2812-3556\end{array}$ & $\begin{array}{c}5 \text { th } \\
\text { 2557-4453 }\end{array}$ & $\begin{array}{c}6 \text { th } \\
4454-5183\end{array}$ & $\begin{array}{c}7 \text { th } \\
5184-5914\end{array}$ & $\begin{array}{c}\text { 8th } \\
5915-6879\end{array}$ & $\begin{array}{c}\text { 6880-8500 } \\
8501-12351\end{array}$ \\
\hline 1970 & 31.35 & 16.32 & 3.72 & 5.60 & 4.52 & 12.14 & 6.35 & 9.75 & 4.71 & 5.53 \\
1980 & 30.99 & 13.12 & 5.84 & 6.97 & 3.96 & 11.31 & 7.85 & 10.45 & 5.63 & 3.88 \\
1990 & 37.14 & 14.64 & 4.01 & 3.50 & 4.66 & 9.01 & 7.55 & 12.46 & 3.17 & 3.85 \\
2000 & 33.24 & 19.27 & 4.44 & 3.61 & 4.50 & 8.46 & 6.35 & 10.42 & 5.43 & 4.28 \\
\hline
\end{tabular}

Source: IMF, Directions of Trade Statistics

We employ the deciles of the distance distribution to filter the (weighted and unweighted) ITN matrices. More specifically, we build two sets of 10 subnetworks to obtain two families of distance-conditioned ITNs. The first family, which we call the cumulated distance-conditioned ITN, is obtained by keeping in the networks, for each decile of the distribution of distance, only the arcs associated to geographical distances smaller than 
the upper-limit of that decile. For example, the 4th cumulated distance-conditioned ITN is obtained by keeping in the binary and weighted matrix only the arcs associated to pairs $(i, j)$ of countries that are located at a distance below that of the upper-limit of the 4th decile of the distribution of distance (i.e. $3556 \mathrm{~km}$ ).

The second family of matrices, called simply distance-conditioned ITN, is obtained by keeping in each one of the ten networks, only the arcs associated to geographical distances belonging to that decile. For instance, the 4th distance-conditioned ITN is obtained by keeping in the binary and weighted matrices only the arcs associated to pairs $(i, j)$ of countries that are located at a distance lower than that of the upper-limit of the 4th decile and above the upper-limit of the 3rd decile of the distance distribution (i.e., between $2811 \mathrm{~km}$ and $3556 \mathrm{~km}){ }^{13}$

More formally, let us label with $\delta_{(1)}, \ldots, \delta_{(10)}$ the deciles (in $\mathrm{km}$ ) of the distribution distance, with $\delta_{(0)}=0$. In each year, we build 10 cumulated distance-conditioned weight matrices, $\mathcal{W}_{k}^{C}=\left\{w_{k}^{C}(i, j)\right\}, k=1, \ldots, 10$, according to the following rule:

$$
\begin{cases}w_{k}^{C}(i, j)=w(i, j) & \text { if } d(i, j) \leq \delta_{(k)} \\ w_{k}^{C}(i, j)=0 & \text { otherwise }\end{cases}
$$

and 10 distance-conditioned matrices $\mathcal{W}_{k}^{N C}=\left\{w_{k}^{N C}(i, j)\right\}$, for $k=1, \ldots, 10$ :

$$
\begin{cases}w_{k}^{N C}(i, j)=w(i, j) & \text { if } \delta_{(k-1)} \leq d(i, j) \leq \delta_{(k)} \\ w_{k}^{N C}(i, j)=0 & \text { otherwise }\end{cases}
$$

Obviously, the two network families are complementary. From the definitions above, one can indeed start from $\mathcal{W}_{k}^{C}$ and remove links in $\mathcal{W}_{k-1}^{C}$ to get $\mathcal{W}_{k}^{N C}$. Similarly, one can build $\mathcal{W}_{k}^{C}$ starting from $\mathcal{W}_{h}^{N C}, h=1, \ldots, k$. Whereas cumulated networks give us a picture of the ITN for all trade relationships between countries that are distant less than a given threshold, distance-conditioned networks (non-cumulated) tell us what trade relationships can be imputed to trade relationships between pair of countries whose distance is within a certain range.

Cumulative and simple distance-conditioned networks are then analyzed both as binary (unweighted) networks and as weighted networks. In the binary case, one simply builds the corresponding binary matrices $\mathcal{A}_{k}^{C}=\left\{a_{k}^{C}(i, j)\right\}$ and $\mathcal{A}_{k}^{N C}=\left\{a_{k}^{N C}(i, j)\right\}$ by adding an arc whenever the correspondent entry in the weighted matrix is positive. It must be noticed that weighted and binary matrices provide complementary information

\footnotetext{
${ }^{13}$ More generally, one can use any quantile-based breakdown of the original distribution range, e.g. quintiles or percentiles. The choice of deciles has been made in order to efficiently trade off the need for a sufficiently larger number of distance classes and a sufficiently large number of observations in each quantile class. Note also that in principle one could have employed distance classes delimited by absolute $\mathrm{km}$ values, independently on the distribution. We preferred to use the quantile-based breakdown because in so doing we are sure that in each class there will be the same, fixed number of links. This implies that each sub-network displays the same density. This avoids comparing sub-networks characterized by different densities.
} 
on the role of different types of costs in international trade. In fact, the presence of fixed costs to access foreign markets should affect the characteristics of the binary matrix, determining the number of links that each country has, whereas trade variable costs (e.g. of the "iceberg" type) should affect links' weights, conditional on a link to be already in place.

In what follows, we start by presenting some baseline results for year 2000. The same exercise is repeated for each decade, from 1970 up to 2000, in order to test whether the impact of distance has changed over the years.

\section{Results}

As customary in this literature (see for example Fagiolo et al., 2009, 2010; De Benedictis and Tajoli, 2011), we begin by analyzing the main topological characteristics of the network. The crucial difference with previous work is that we do so across the different subnetworks obtained above by conditioning to distance deciles. In particular, we focus on network connectivity (i.e. whether any two nodes can be connected in the network by a chain of links), the distributions of total node degree (i.e. a country's number of partners) and total node strength (i.e., a country's total imports plus exports), average nearestneighbors degree and strength (i.e., average total degree or strength of the partners of a country), and clustering (i.e. the probability, possibly weighted by link weights, that any pair of partners of a node are themselves partners). Furthermore, for each decile, we examine the distribution of link weights, the correlation structure among node statistics, and the correlation structure between node statistics and some country macroeconomic characteristics (e.g., GDP and GDP per capita).

The analysis of subnetworks created for different distance deciles shows that considering the geographical distance between the nodes of the network indeed matters. Distanceconditioned trade subnetworks display topological properties that greatly change with distance deciles, as discussed in more details below.

\subsection{Connectivity in the ITN}

To begin with, we explore connectivity of the ITN as distance changes. The overall ITN is evidently connected, i.e. any country in the world can be reached from anywhere else in the network through undirected trade links. ${ }^{14}$ When splitting the network based on distance, two interesting connectivity statistics are the number of connected components and the size of the giant component (i.e. the number of nodes making up the largest subset of connected nodes in the network). Of course if only one connected component is

\footnotetext{
${ }^{14}$ We employ thorughout the concept of weak connectivity, which considers any two nodes connected if there is any link between them, irrespective of its directionality. Strong connectivity instead requires that pairs of nodes can be reacheable via a directed path.
} 
observed then the giant component has the same size of the overall network (as happens for the ITN as a whole).
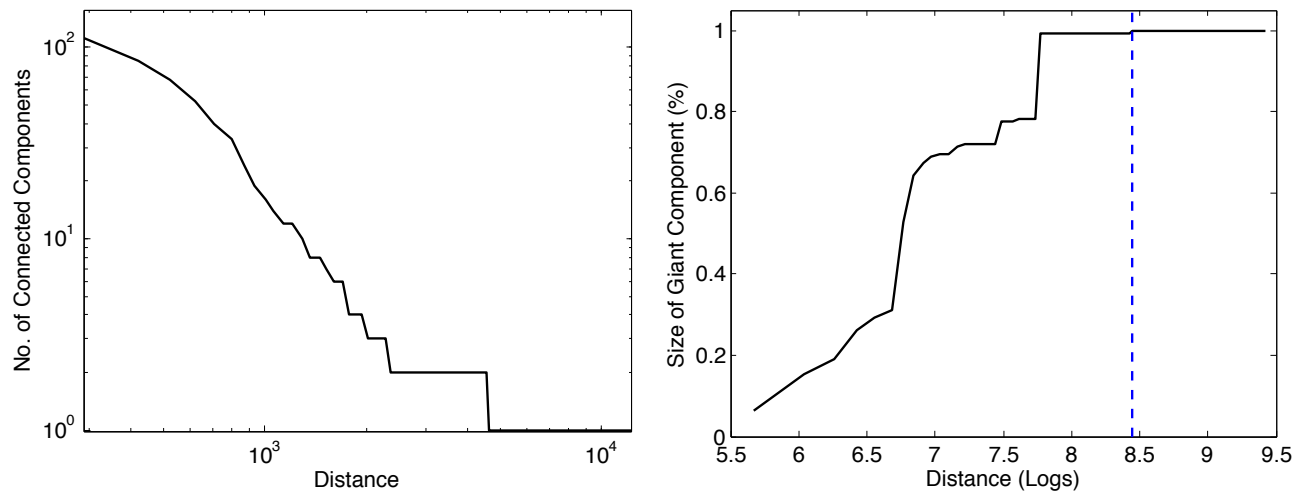

Figure 2: Connectivity in distance-conditioned cumulated ITNs in year 2000. Left: Number of connected components vs. distance (log-log scale). Right: Size of the giant component (as a share of number of nodes) vs. distance.

Figure 2 shows how connectivity statistics change with distance in cumulated ITNs for year 2000. As we keep adding longer distance trade relationships in the network, the number of connected components sharply decreases, following a power-law shape. Initially, when only small-distance trades are taken into account, a very large number of small components emerge. This means that at small distances the ITN is extremely disconnected. Indeed, the hubs of the network are typically joined with countries that lie far apart. Accordingly, the relative size of the giant component is very small (about $7 \%$ ) and quickly increases towards 1 as we consider longer-distance relationships. Complete connectivity only emerges when we start taking into the picture trade relationships occurring at $4000 \mathrm{~km}$ or more. This allows one to have in the network also links between the hubs and peripheral countries that are only connected to the hubs. ${ }^{15}$

\subsection{Link weights and strength}

Connectivity of the ITN over different distance intervals may be also studied in terms of link weights, and node degree and strength. Fig. 3 shows that as we consider longerdistanced trade relationships in the ITN, average link weight initially decreases very rapidly and linearly, as predicted by the gravity equation. At higher distances, the effect of distance becomes strongly non-linear with respect to the logs of link weights, and in fact the average weight value increases for intermediate distances and then decreases again. All that maps into a smoother pattern for cumulated distance-conditioned networks: the

\footnotetext{
${ }^{15}$ This result is very much consistent over the years. Indeed, when looking at simple distanceconditioned ITNs, one typically observes a stable number of connected components across deciles (between 15 and 23) and a few of isolate nodes (between 4 and 13).
} 
steepness of the relationship between average link-weight decreases and distance decreases as we reach the median of the distribution.

Note also that the variance of link weights (not shown) follows a U-shaped pattern with respect to geographical distance, with the highest variance displayed at very low and very high distances. Taken together, this evidence confirms the very well-known negative relationship between trade flows and distance stressed in the empirical gravity literature, but highlights very marked non-linearities in the way in which distance affects both the average of (logs of) trade-flows and their conditional variance, especially for long-distance trade relationships.
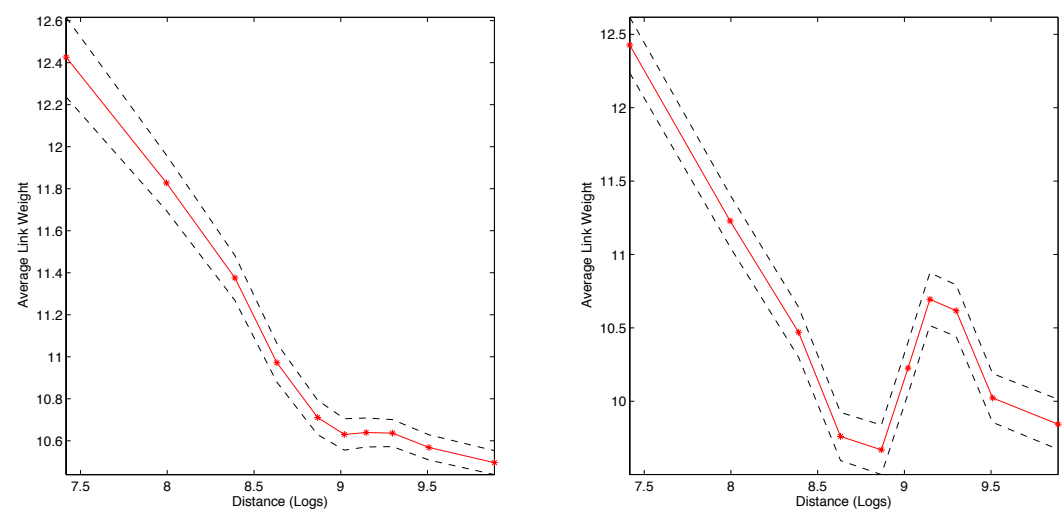

Figure 3: Average link weights (solid lines) for simple (right) and cumulated (left) distanceconditioned networks. Dotted lines: 95\% confidence bands. Year: 2000.

We now turn to study how the correlation between node-specific network properties changes with geographical distance. Figure 4 shows the linear correlation coefficient computed between node out-degree (the number of countries to which a country exports) and out-strength (i.e total exports), with 95\% confidence intervals, in year 2000 for distanceconditioned networks.

As expected, correlation coefficients are very high at all geographical distances: in general terms, countries that trade more also hold more trade partners, irrespective of how far they are located in the world and of which export market we consider. This evidence confirms that the geographical extensive margin and the intensive margins of trade are highly correlated. What is less expected is that distance does not affect significantly this high correlation: if any, a weak positive impact of distance on degree-strength correlation is detected, implying that the relation between number of trade partners and trade flows is marginally stronger for long-distance trade pairs. Following the line of thought discussed in Section 2, this evidence suggests that the relative relevance of fixed and variable costs in affecting overall trade costs - assuming that these are the variables affecting export volumes and the number of partners - is fairly constant across distance. 

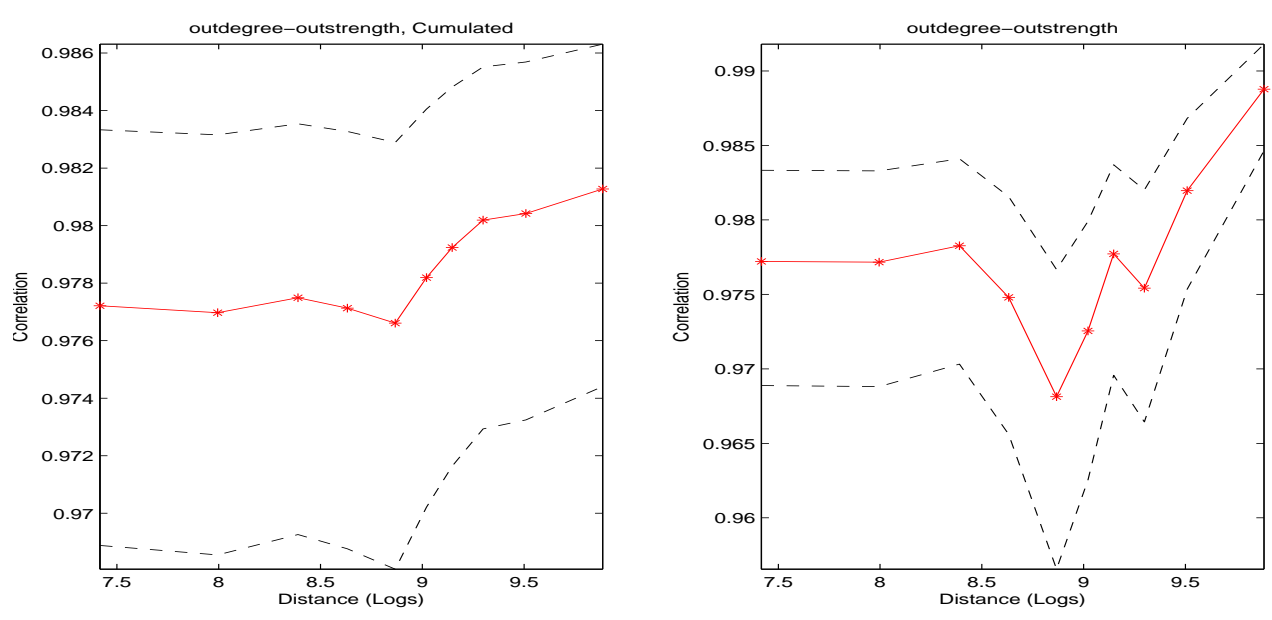

Figure 4: Correlation coefficients (solid lines) between node degrees and node strengths for simple distance-conditioned networks. Dotted lines: 95\% confidence bands. Year: 2000.

\subsection{Assortativity and Clustering}

A number of previous analyses found a marked disassortativity pattern characterizing the ITN, both at the binary and weighted level (Garlaschelli and Loffredo, 2004, 2005; Serrano and Boguná, 2003; Fagiolo et al., 2008, 2009). A disassortative (assortative) network is a graph where there is a negative (positive) correlation between node degrees/strengths and average nearest-neighbor degrees/strengths. More generally, a disassortative network is one where more (and more strongly) connected nodes are typically connected with less (and less strongly) connected nodes, i.e. countries tend to be connected with partners that are different in terms of connectivity. But do disassortativity patterns in the ITN depend on geographical distance? For example, do countries who trade more at a given distance tend to trade with partners that trade less at the same distance scale?

To answer these questions, we analyze the existing correlation structure between a country's out-degree (or out-strength) and its average nearest-neighbor out-degree (outstrength) in the distance-conditioned sub-networks obtained from our ITN. More precisely, in the binary network, we correlate the number of countries which a country exports to (its out-degree), with the average number of countries which those partners exports to (its degree out-out). The same intuition applies for the weighted network, once node degree is replaced by node strength. Figure 5 summarizes our results in year 2000, for cumulated vs. simple distance-conditioned networks, and for binary vs. weighted descriptions.

First of all, our analysis confirms that the aggregate ITN is found to be disassortative at the aggregate level: the correlation between node degree (strength) and ANND (ANNS) is negative in the cumulated network including all distances (left panels of Figure 5). However, if one conditions the correlation structure to geographical distance, it is easy to see that short-distanced networks exhibit a very assortative pattern. The 
correlation coefficient is positive and quite high for all the short-distance networks displayed in the figure, and the pattern is very similar in the binary and weighted cases. As we add to the ITN links associated to higher distances, correlation coefficients decrease smoothly and non-linearly towards a disassortative pattern. More specifically, as Figure 5 shows, the ITN displays an assortative pattern for small distances, it becomes weakly disassortative for long-distance partnerships, while at intermediate distance no clear correlation patterns emerge. This means that, when only short-distance trade partnerships are considered, countries with many partners tend to trade with countries holding many connections. Conversely, at high distances, very connected countries typically trade with poorly connected partners ${ }^{16}$
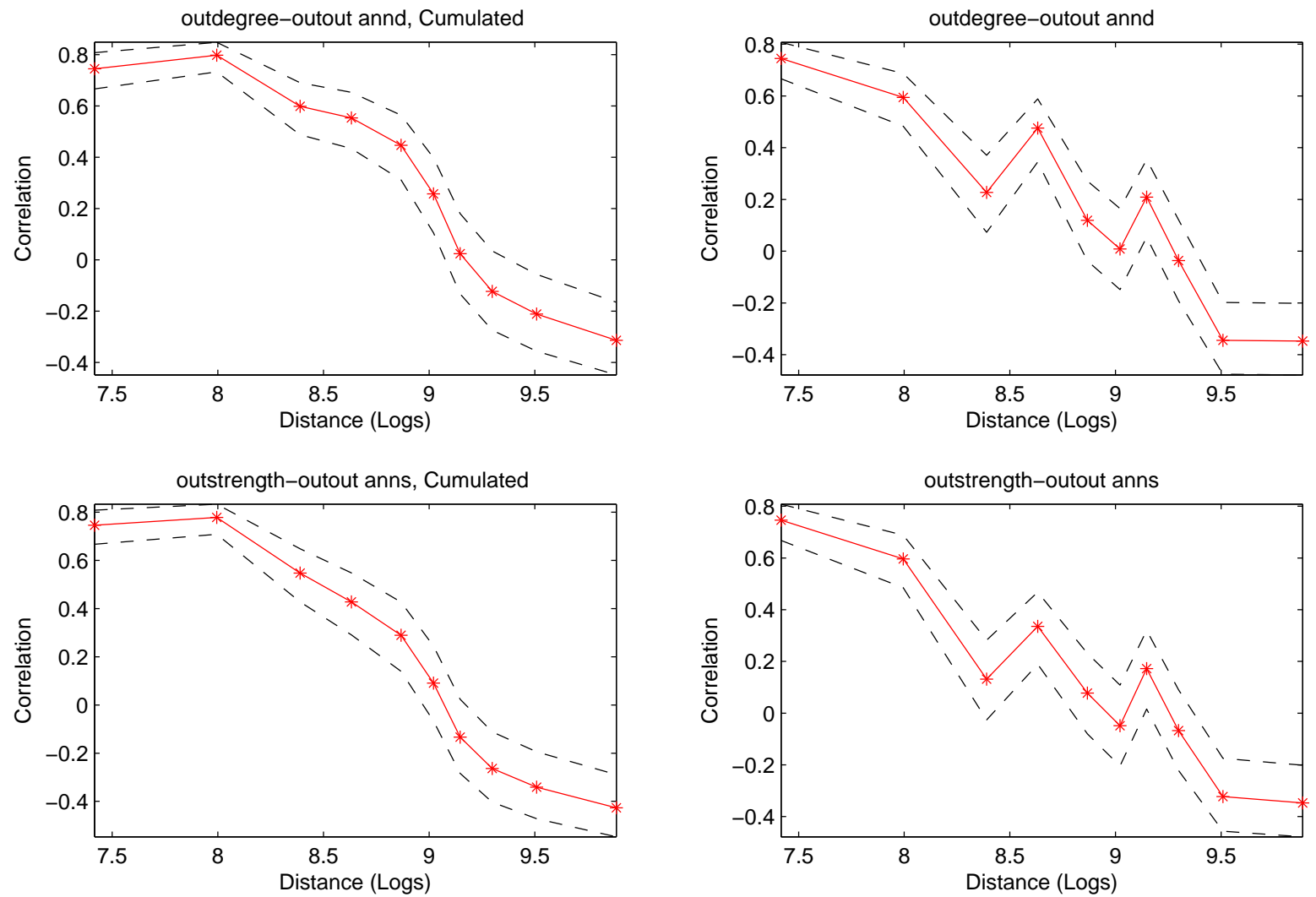

Figure 5: Left: Assortativity in cumulated distance-conditioned directed networks. Top: The binary ITN case; bottom: The weighted ITN case. Right: Assortativity in simple distanceconditioned directed networks. Top: The binary ITN case; bottom: The weighted ITN case. For the binary case the correlation coefficient is between node out-degree and directed average nearest-neighbor degree (annd) measures. For the weighted case the correlation coefficient is between node out-strength and directed average nearest-neighbor strength (anns) measures. Dotted lines: 95\% confidence bands. Year: 2000.

Taken together, this evidence implies that patterns of assortativity or disassortativity

\footnotetext{
${ }^{16}$ Note that we find a very similar pattern also when disaggregating assortativity with respect to import market distance (as mentioned, the results for the analysis on imports are not reported in this paper), suggesting that the directionality of trade flows is not a crucial factor in determining this result.
} 
in the ITN are strongly dependent on distance, and the marked overall disassortativity of the ITN is mainly driven by high-distance trade relationships. A number of factors could produce this result. On the one hand, the existence of many preferential regional trade agreements (RTAs) (WTO, 2011) fostering trade between similar and often neighboring countries can explain the assortativity found at short distances. Regional trade agreements will typically generate a high number of strong trade links for all the member countries within the region, producing the positive correlation result. On the other hand, countries that are geographically isolated from the rest of the world, when choosing an export market for their goods might tend to prefer a well-connected country that works as a hub to connect them to rest of the system.

This preference will give rise to a disassortativity pattern at long distances. In the thirty-year period considered by our analysis, the assortativity patterns discussed so far seem to be relatively robust. If any, one notices an increasing disassortativity over time at higher distances. In other words, countries with a high out-degree/out-strength, i.e. important exporters, have been experiencing a significantly higher probability of being connected with countries that in turn export relatively little to a limited number of markets. By year 2000, the largest exporters had reached nearly every market in the world, even the countries that appear geographically isolated. Therefore, the marginal cost of accessing additional markets seem to have become very small, especially for big exporters.

We turn now to explore the observed patterns of distance-conditioned clustering coefficients. The clustering coefficient of a node in the ITN measures the likelihood that a country forms intensive-trade triangles with its trade partners (Fagiolo, 2007). Previous studies show that the binary version of the ITN (at all distances) is highly clustered, whereas the weighted ITN displays a relatively weaker clustering, due to the presence of many low-trade interactions that weaken the ex-post intensity of the many triangular trade relations existing in the ITN (Fagiolo et al., 2009).

Figure 6 shows that distance plays a crucial role also in explaining evidence on clustering. Indeed, both binary (BCC) and weighted (WCC) clustering coefficient in cumulated distance-conditioned ITNs (left panels of the figure) non-linearly increase as we add to the network longer distance trade partnerships. More specifically, BCC and WCC reach a maximum when we consider trade relations close to $3800 \mathrm{~km}$, and then decrease as we approach $6000 \mathrm{~km}$, and then increase again towards the absolute maximum of average clustering. This suggests that most of the contribution to maximum clustering by binary and weighted triangular trade interactions comes from smaller-distance trade flows. This is confirmed by looking at the plot of the BCC and WCC for simple distance-conditioned ITNs (right panels). Average clustering non-linearly decreases towards zero as distance increases, meaning that triples of countries that are very distant to each other almost never engage in triangular trade relationships (with the exceptions of trade flows occur- 

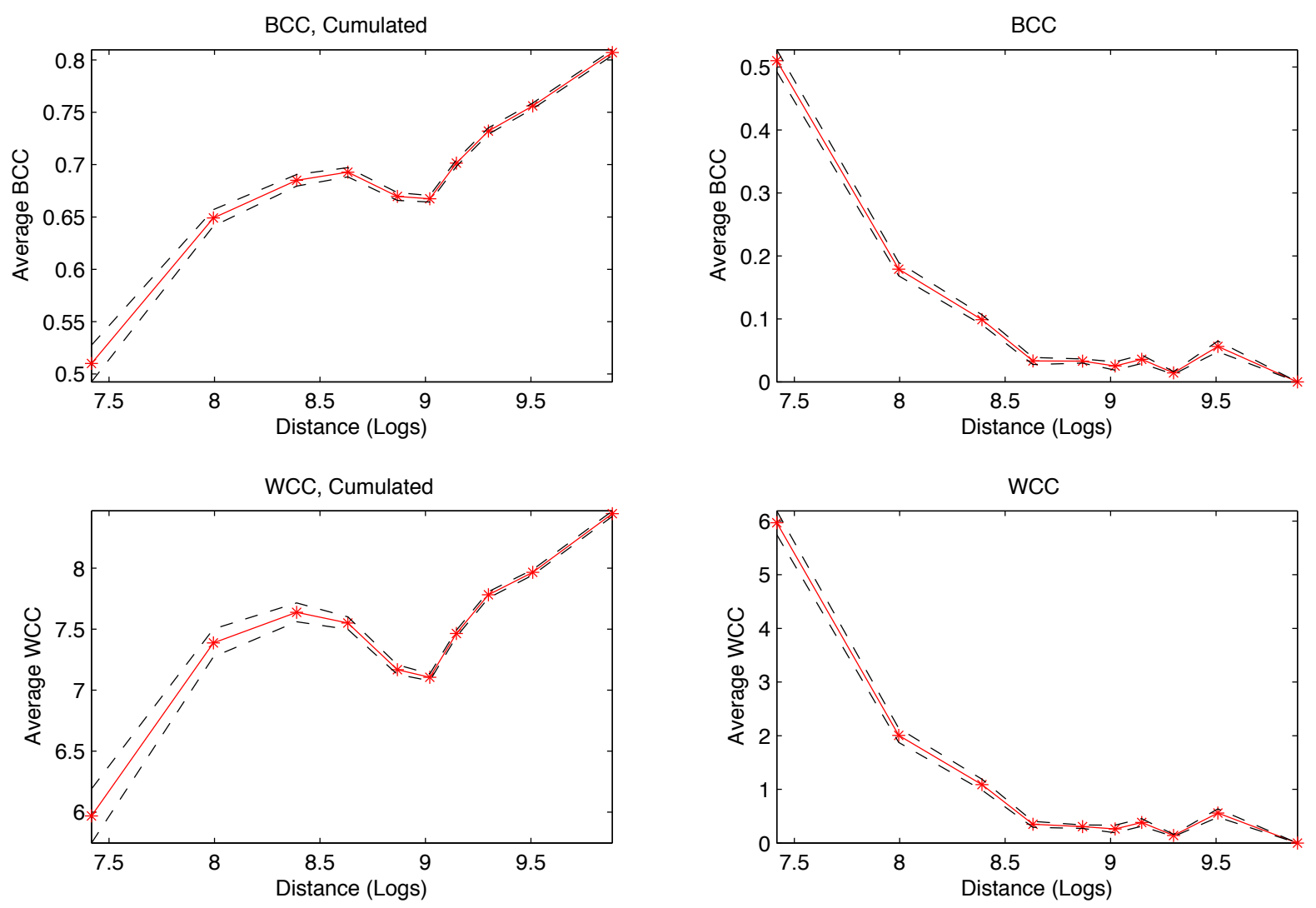

Figure 6: Left: Clustering in distance-conditioned directed networks. Right: Clustering in simple distance-conditioned directed networks. Top: the binary ITN case; bottom: the weighted ITN case. For the binary case the binary clustering coefficient (BCC) computes for each node the percentage of closed triangles in the node's neighborhood. In the weighted case, the weighted clustering coefficient (WCC) computes the intensity of such closed triangles, where triangle edges are weighted by link weights. Dotted lines: 95\% confidence bands. Year: 2000. 
ring between countries whose distance is about $9900 \mathrm{~km})$. Hence, only short-distanced triples of countries contribute to the large value of the overall $\mathrm{BCC}$ and $\mathrm{WCC}$ found in the ITN. Notice again that strong non-linear effects affect the link between logs of distance and network statistics.

Overall, also the emergence of a sharp decreasing relationship between distance and clustering can be due to the impact of RTA effects: a RTA indeed favors clustering as it creates and enforces the establishments of cliques (and thus triangular trade relation-

ships) among countries located relatively close to each other. Instead, the low clustering coefficient found for networks including only countries that are geographically far apart reinforces the idea that some countries may play the role of hubs of the system.

\subsection{The role of country size and income}

Gravity models emphasize the role played by economic size and income, in addition to geographical distance, in shaping bilateral trade flows. To address this issue in the present context, we begin by examining correlation patterns between network-based statistics and country size and income (as measured by GDP and GDP per capita) over different distance deciles. It is in fact well-known that - everything else being equal- large countries tend to be also large traders.

The four panels of Figure 7 show for year 2000 the plots of linear correlation coefficients between node degree/strength and node GDP/per capita GDP, conditioned on distance deciles.

We note that the correlation between GDP and total degree and strength is generally positive, even if it varies a lot across distance deciles. The correlation is especially high for a subset of trade flows involving pairs of countries that lie very far apart (between $6000 \mathrm{~km}$ and $7000 \mathrm{~km}$ ), but do not belong to the last two deciles. This is true also for per-capita GDP, and it means that at large distances economic size and income of countries heavily and positively influence their trade shares. Note also that correlation coefficients first increase and then decrease when country GDP is considered. While distance certainly affects trade flows, the observed pattern indicates the extent of this influence depends also on countries' economic size. These results suggest that there could be a sort of optimal distance value that maximizes the correlation between country size and country connectivity in terms of trade partners and total trade. On the contrary, at some distances, the correlation between node connectivity and per-capita GDP could be very weak, or not-significantly different from zero, as shown in the figure for a distance around $4000 \mathrm{~km}$.

The foregoing size/income correlation analysis, however, does not take into account the fact that export flows bilaterally depend on both origin and destination market sizes, as gravity model estimates of trade always confirm. In order to allow for such a depen- 

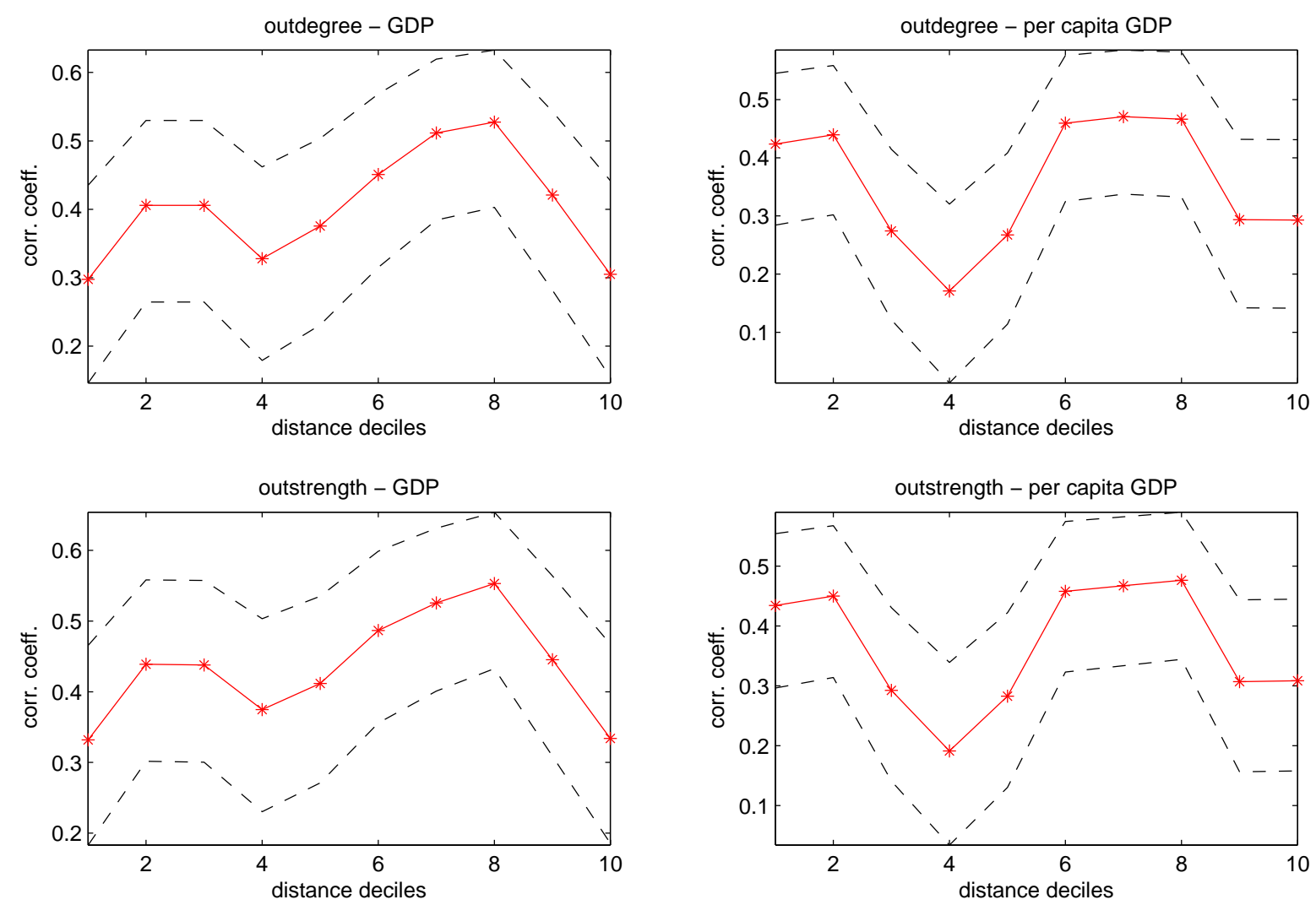

Figure 7: Correlation between node degree/strength and GDP/per capita GDP vs. distance in year 2000. Dotted lines: $95 \%$ confidence bands. 
dence in our data, we have investigated what happens when one re-scales link weights by the expected bilateral flow in a frictionless world. In this setup, the $i j$ link weight is simply defined as exports from $i$ to $j$ (in levels) divided by the product between the GDPs of country $i$ and country $\mathrm{j}$. In this way, the impact of origin and destination market sizes are washed away and we can understand how distance affect the properties of the ITN regardless of any size effect.

As we did above, we used the logs of rescaled link weights, namely the log of exports minus the sum of the logs of $i$ and $j$ GDPs. In order to avoid negative weights, we have translated the entire distribution by a minimum threshold, in such a way to preserve existing density. We have re-computed all (weighted) topological properties of this GDPrescaled ITN to see how they change across the deciles of the logs of distance distribution. Of course the binary version of the ITN is almost unaffected by this change, as the rescaling only influences positive original weights.

Results strongly confirm the main insights coming from the foregoing analysis. For example, GDP re-scaling preserves the negative relationship between link weights and distance in the distance-conditioned networks. What is more, non-linearities still emerge in the log-log relation between weights and distance. The correlation between node degree and node strength remains positive and very high, and relatively less sensitive to distance. Weighted disassortativity patterns are instead unaffected by the rescaling. This means that this result is not driven by country economic sizes. Finally, the increasing relation between average weighted clustering and distance in cumulated distance-conditioned networks is still present, even if slightly less important: when we account for country GDPs, distance seems to impact a little less on clustering. This difference wades away in simple distance-conditioned networks, where we still observe a significant and negative impact of distance on average weighted clustering.

This analysis can be of course extended by more strongly pursuing the idea of filtering away gravity-based influences on export flows. Following Fagiolo (2010), one could think to fit a gravity model to bilateral trade flows and to employ the residuals to build a trade network where now flows are net of any effect coming from size, borders, trade unions, etc. (excluding distance), and to explore the properties of such network as distance changes.

\subsection{Network structure and distance over time}

So far, we have explored the connection between geographical distance and network structure by focusing on year 2000. But what happens to this connection over the years?

The distribution of average link weights over distance shown in Figures 8 and 9 displays some similarities over time, but also some changes: the average link weight presents a downward trend over distance in all years, but the slope and the kinks are different. In particular, in 1970 and in 1980 the weight of trade links moves irregularly over a wide 
range of intermediate distances. In the more recent decades, some of these swings smooth out, but the increase in the link weight at middle-high distances becomes more evident. Overall, both from a simple and cumulated perspective, the relation between trade flows and distance was very non-linearly shaped also in the past, with only small-distanced and large-distanced trade flows markedly decreasing with geographical distance.
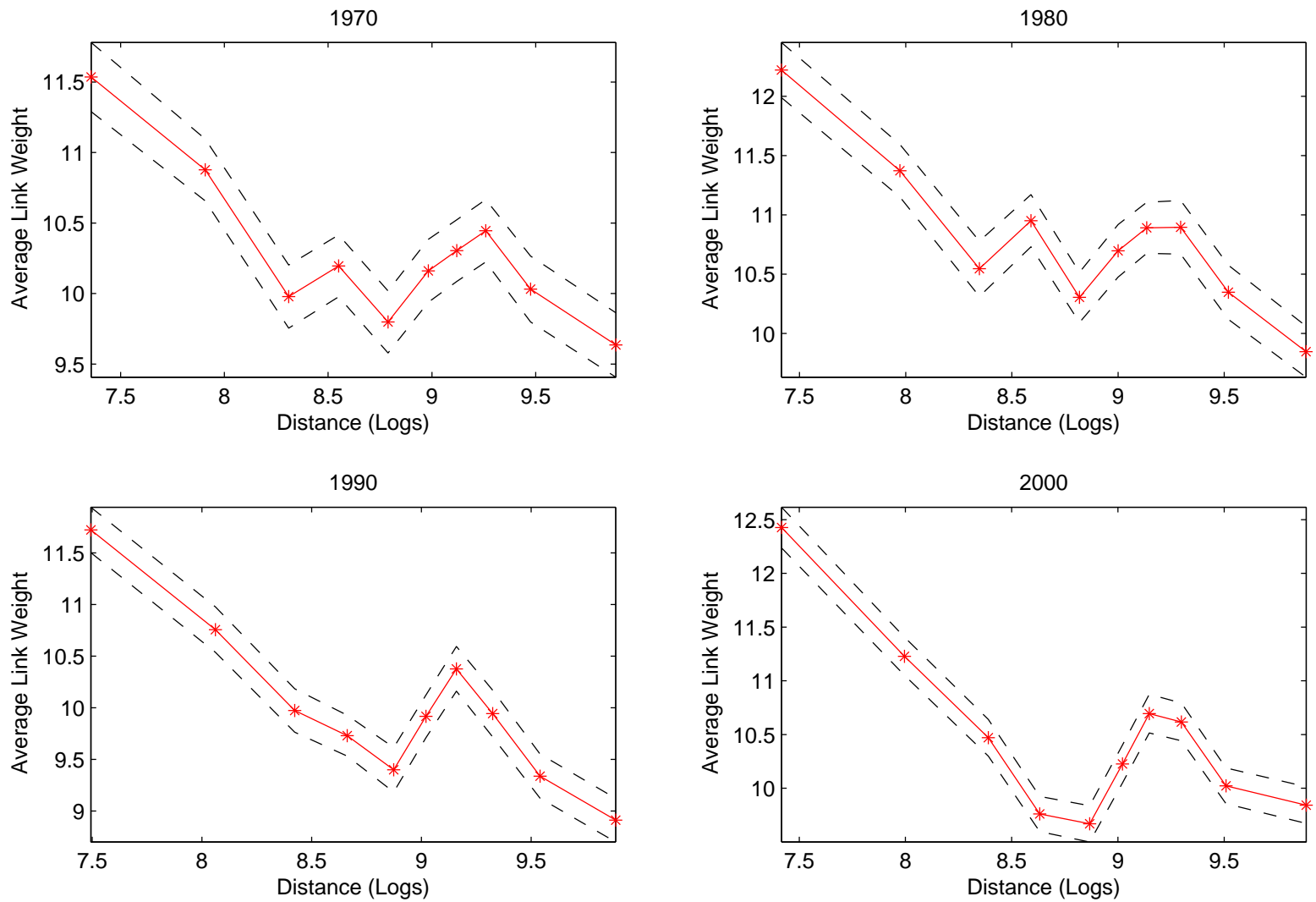

Figure 8: Average link weights (solid lines) for simple distance-conditioned networks. Dotted lines: 95\% confidence bands. Years: 1970, 1980, 1990, 2000.

Correlations between node degree/strength and country size/income across distance follows a very similar pattern from 1970 to 2000, basically reproducing what we observe in Figure 7. The persistence in the role of distance that we pick up in time is in line with the results found elsewhere in the literature using the gravity model of trade (Disdier and Head, 2008). In our framework, we can interpret this result by arguing that, as the size of the network increases over time because the number of relevant trading countries increases, geographical distance continues to matter, as variable costs per kilometer might decline, but overall trade costs to stay connected with the entire network remain high.

Our analysis shows that while the role of distance did not decline in time, its impact on the relation between country's economic size or income and country connectivity has somewhat changed. In fact, we observe that the correlation levels between nodes' strength and countries' average income were higher in 1970 than in 2000. This hints to an increased participation to global trade, especially by low and middle-income countries, probably 

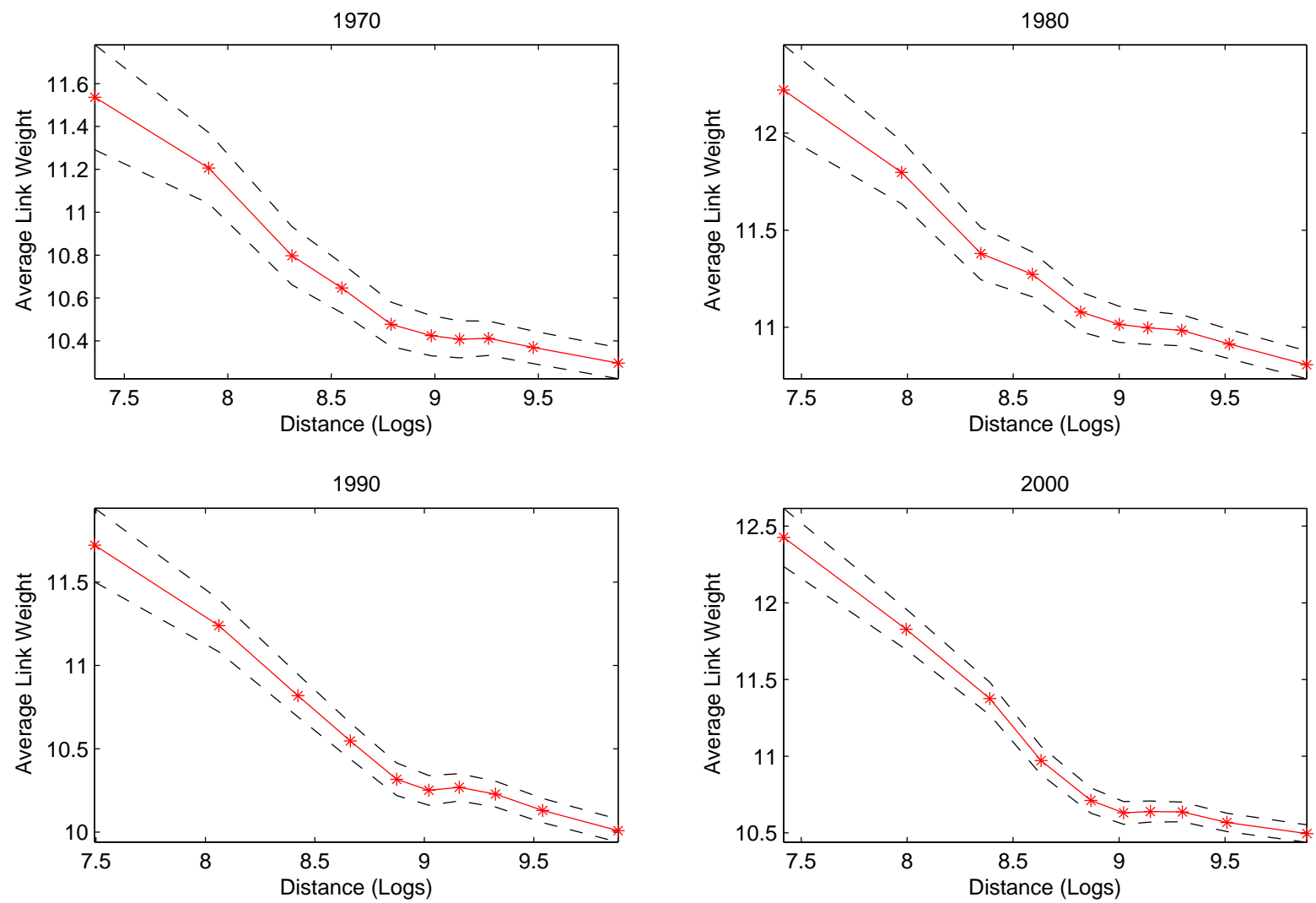

Figure 9: Average link weights (solid lines) for cumulative distance-conditioned networks. Dotted lines: 95\% confidence bands. Years: 1970, 1980, 1990, 2000. 


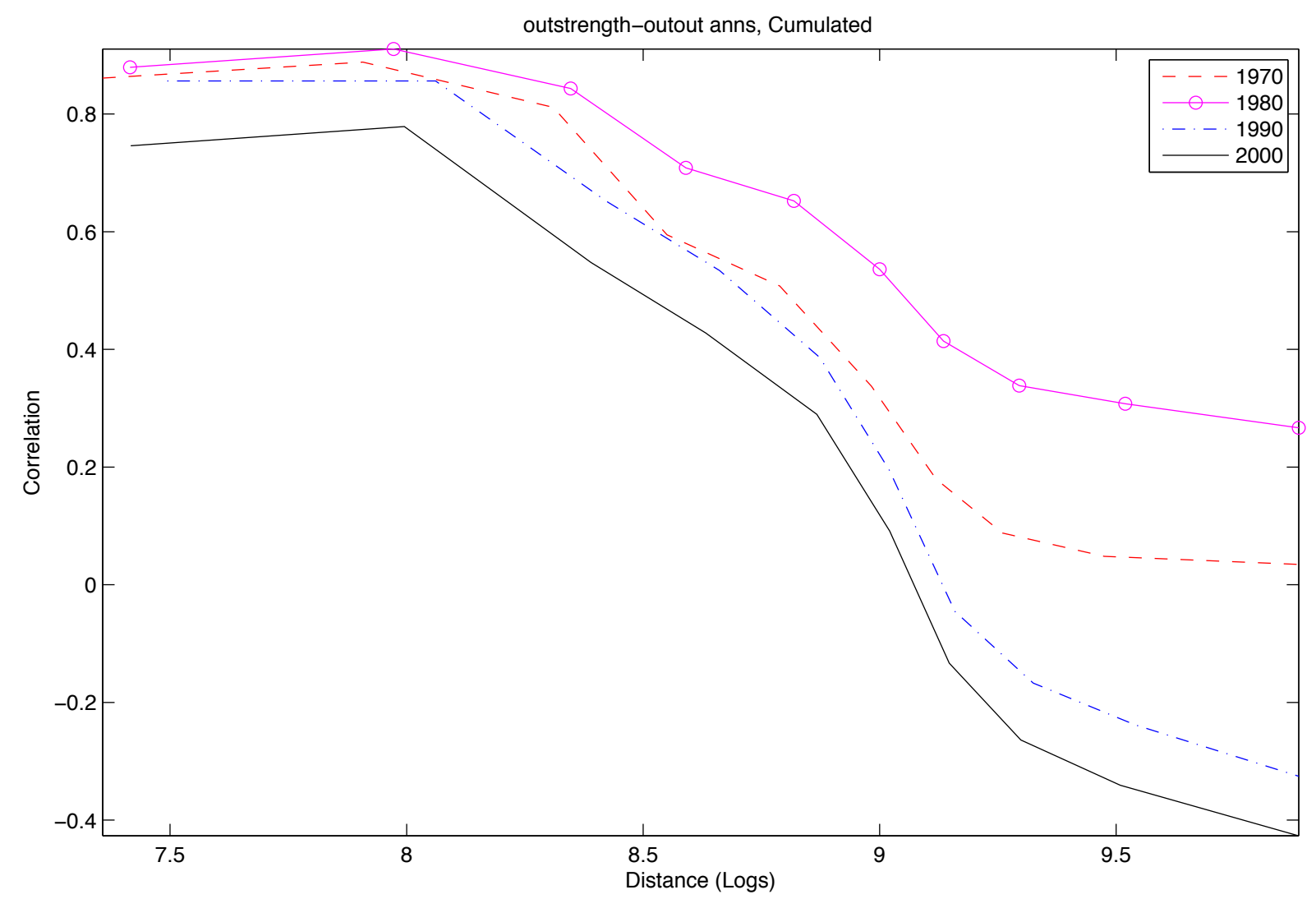

Figure 10: Assortativity and disassortativity. Years: 1970, 1980, 1990, 2000.

due to the secular decline in trade costs and in other trade barriers.

The reduction in the correlation seems to be the result of a change of the ITN affecting especially countries in middle-distanced subnetworks: for these countries the correlation between their trade flows and average incomes has become lower and less significant, possibly because of the historical reduction in trade costs. Instead, as distance increases beyond this middle range, trade costs are still relevant and therefore higher incomes - capable of overcoming such trade costs - are still correlated with higher trade flows.

Much more evident are the changes over time in other features of the network structure along different distances. As shown in Figure 10, the property of being assortative at short distances but disassortative (or at least much less assortative) at large distances has characterized the ITN since the 1970s. But over time, the extent of the disassortativity at longer distances has increased substantially. In the past, when the potential number of trading countries was smaller and trade costs were higher, the tendency to link to similar countries was stronger, and overall assortativity prevailed. Nowadays, as the heterogeneity of countries involved in international trade has increased, the disassortativity for large-distanced countries is much higher.

Also clustering has increased remarkably over the years, even if the pattern over distance is somewhat similar in time (see Figure 11). This can again be related to increase 


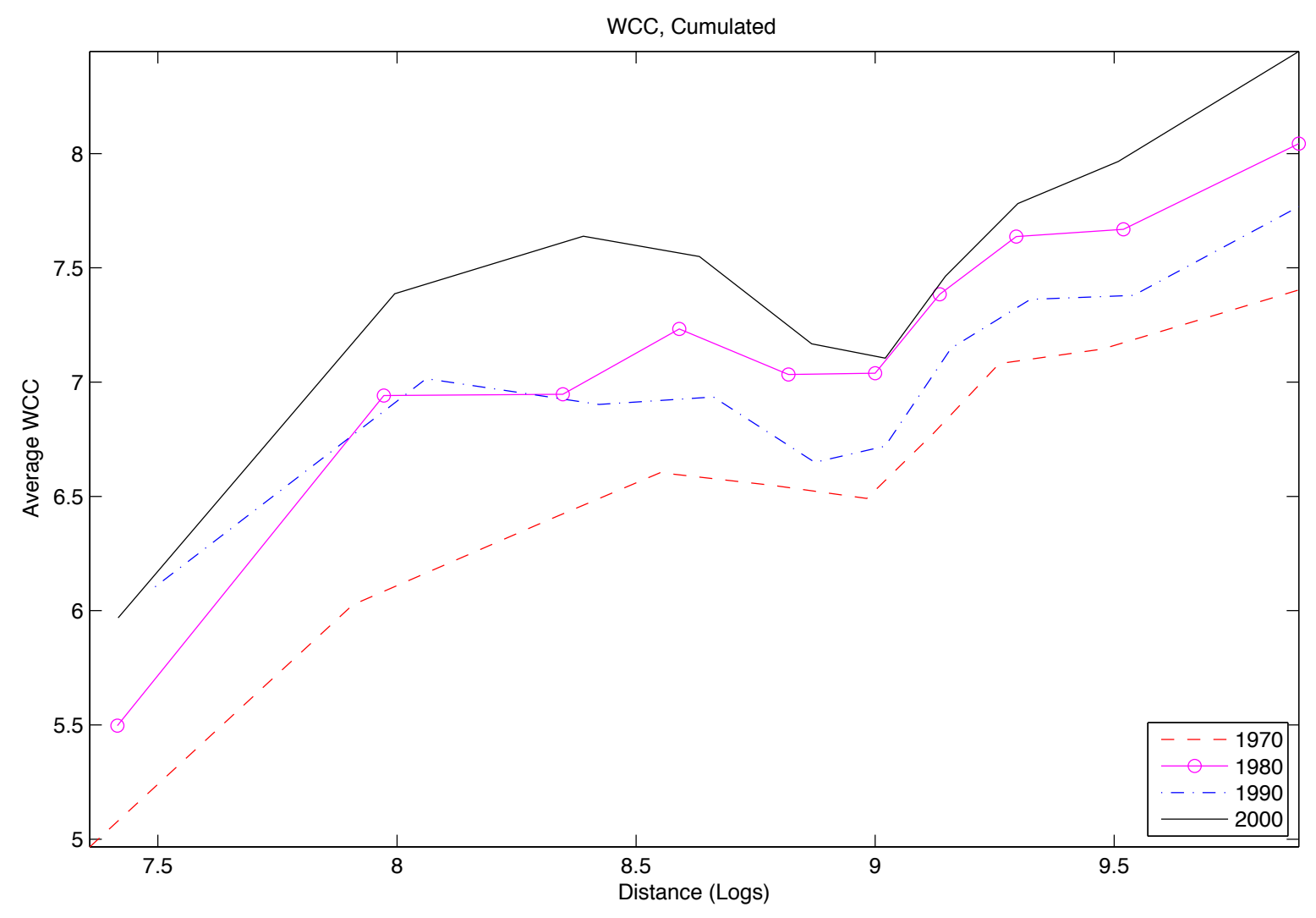

Figure 11: Clustering. Years: 1970, 1980, 1990, 2000.

in the number of preferential trade agreements signed between countries. Interestingly, the figure shows a clear increase in the clustering coefficient also for far away countries. This evidence is in line with the more recent tendency of countries to sign preferential trade agreements not so much with neighboring partners, but also with countries in other continents (see WTO, 2011). These results confirm that even if globalization and technological improvements have not eliminated the role of distance as a hindering factor to trade, its impact on the overall structure of the ITN has changed over the years.

\section{Conclusions}

In this paper we explored whether the topological architecture of the International Trade Network (ITN) changes in geographical space and along time. We employed geographical distance between countries in the world to filter trade relationships in the ITN to build sub-networks of countries who trade with partners located at similar geographical distances.

Our main result is that geographical distance matters also from a complex network perspectives. Furthermore, we show that the effect of distance on the ITN topological properties is highly nonlinear. 
Our results highlight that the role of distance and trade costs in affecting trade patterns is not only based on linear bilateral distance in miles, and that the relevance of both a fixed and variable component of trade costs can jointly generate the type of non-linearity observed here.

The analysis also shows that the geographical pattern of trade and the geographical extensive margin are very different for groups of countries at different distance ranges: while the aggregated ITN over all distance ranges is disassortative, shorter distance subnetworks are assortative. Furthermore, the trade intensity and number of triangular trade relationships decreases as distance increases. Both results confirm that the structure of the ITN changes dramatically in geographical space.

The structure of the network shows also the role of specific countries working as hubs of the system. These results indicate that the role of distance is different for countries with different economic size, which should have a different capacity to overcome the trade costs that distance imply. Large countries with very high trade volumes can exploit the economies of scale associated with their size created by the presence of fixed costs. Therefore, they can trade profitably also with far-away countries, and therefore they can play the role of hubs of the system.

Comparing the results of the analysis over time we note that the average trade partner in the middle-distanced group has increased its relative strength or share of total trade between 1970 and 2000, hinting to an increase participation in international trade of such a group of countries. This is confirmed by the correlation structure between network statistics and per-capita GDP.

The effect of distance over time is not trivial. Over the time period examined, the size of the network increases, as the number of connected countries increase, and the diameter of the network grows. Therefore distance remains relevant (as shown also in gravity models), in spite of a (relative) decline in trade costs, because the length and number of trade links has increased.

This study can be extended in many ways. First, one may want to explore the topological properties of the ITN by explicitly embedding the network in a spatial structure and use methodologies developed in the literature on spatial networks (Barthelemy, 2011). Second, a more theoretical explanation building on the interplay between fixed (sunk) and variable trade costs may be conceived, so as to develop a proper network formation model able to replicate, for example, the structural breaks detected in e.g. assortativity patterns when we move from smaller to higher distances. 


\section{References}

Abeysinghe, T. and K. Forbes (2005), "Trade linkages and output-multiplier effects: a structural VAR approach with a focus on Asia", Review of International Economics, 13: $356-375$.

Albert, R. and A.-L. Barabási (2002), "Statistical Mechanics of Complex Networks", Review of Modern Physics, 74: 47-97.

Anderson, J. E. (2011), "The gravity model", Annual Review of Economics, 3: 133-160.

Anderson, J. E. and E. van Wincoop (2003), "Gravity with Gravitas: A Solution to the Border Puzzle", American Economic Review, 93: 170-192.

Anderson, J. E. and E. van Wincoop (2004), "Trade Costs", Journal of Econmic Literature, 42: 691-751.

Anderson, J. E. and Y. V. Yotov (2012), "The gold standard gravity", NBER working papers, No. 17835, .

Artis, M., A.-B. Galvão and M. Marcellino (2003), "The transmission mechanism in a changing world", Economics Working Papers ECO2003/18, European University Institute.

Baltagi, B. H., P. Egger and M. Pfaffermayr (2007), "Estimating models of complex FDI: Are there third-country effects?", Journal of Econometrics, 140: 260-281.

Barigozzi, M., G. Fagiolo and D. Garlaschelli (2010), "The Multi-Network of International Trade: A Commodity-Specific Analysis", Physical Review E, 81: 046104.

Barthelemy, M. (2011), "Spatial Networks", Physics Reports, 499: 1-101.

Bernard, A., J. Jensen, S. Redding and P. Schott (2007), "Firms in international trade", Journal of Economic Perspectives, 21: 105-130.

Bhattacharya, K., G. Mukherjee and S. Manna (2007), "The International Trade Network", in A. Chatterjee and B. Chakrabarti, (eds.), Econophysics of Markets and Business Networks, Springer-Verlag, Milan, Italy.

Bhattacharya, K., G. Mukherjee, J. Sarämaki, K. Kaski and S. Manna (2008), "The International Trade Network: Weighted Network Analysis and Modeling", Journal of Statistical Mechanics: Theory Exp. A, 2: P02002.

Brun, J.-F., C. C., G. P. and de Melo J. (2005), "Has distance died? Evidence from a panel gravity model", World Bank Economic Review, 19: 99-120. 
Chaney, T. (2008), "Distorted Gravity: The Intensive and Extensive Margins of International Trade", American Economic Review, 98: 1707-1721.

Chen, M. X. and S. Joshi (2010), "Third-country effects on the formation of free trade agreements", Journal of International Economics, 82: 238-248.

Coe, D. T., A. Subramanian and N. T. Tamirisa (2007), "The Missing Globalization Puzzle: Evidence of the Declining Importance of Distance", IMF Staff Papers, 54: $34-58$.

De Benedictis, L. and D. Taglioni (2011), "The Gravity Model in International Trade", in L. De Benedictis and L. Salvatici, (eds.), The Trade Impact of European Union Preferential Policies,chapter 4, Springer Berlin Heidelberg, 55-89.

De Benedictis, L. and L. Tajoli (2011), "The World Trade Network", The World Economy, 34: $1417-1454$.

Dees, S. and A. Saint-Guilhem (2011), "The role of the United States in the global economy and its evolution over time", Empirical Economics, 41: 573-591.

Disdier, A. and K. Head (2008), "The Puzzling Persistence of the Distance Effect on Bilateral Trade", Review of Economics and Statistics, 90: 37-48.

Dorogovtsev, S. and J. Mendes (2003), Evolution of Networks: From Biological Nets to the Internet and $W W W$, Oxford, Oxford University Press.

Duenas, M. and G. Fagiolo (2011), "Modeling the International-Trade Network: A Gravity Approach", ArXiv:1112.2867v1 [q-fin.GN], http://arxiv.org/abs/1112.2867.

Eaton, J. and S. Kortum (2002), "Technology, Geography, and Trade", Econometrica, 70: 1741-1779.

Egger, P. and M. Larch (2008), "Interdependent preferential trade agreement memberships: An empirical analysis", Journal of International Economics, 76: 384-399.

Fagiolo, G. (2007), "Clustering in Complex Directed Networks", Physical Review E, 76: 026107.

Fagiolo, G. (2010), "The International-Trade Network: Gravity Equations and Topological Properties", Journal of Economic Interaction and Coordination, 5: 1-25.

Fagiolo, G., S. Schiavo and J. Reyes (2008), "On the topological properties of the world trade web: A weighted network analysis", Physica A, 387: 3868-3873.

Fagiolo, G., S. Schiavo and J. Reyes (2009), "World-trade web: Topological properties, dynamics, and evolution", Physical Review E, 79: 036115. 
Fagiolo, G., S. Schiavo and J. Reyes (2010), "The Evolution of the World Trade Web: A Weighted-Network Approach", Journal of Evolutionary Economics, 20: 479-514.

Feenstra, R. (2002), "Border Effects and the Gravity Equation: Consistent Methods for Estimation", Scottish Journal of Political Economy, 49: 491-506.

Forbes, K. (2002), "Are trade linkages important determinants of country vulnerability to crises?", in S. Edwards and J. A. Frankel, (eds.), Preventing Currency Crises in Emerging Markets, Chicago, University of Chicago Press.

Garlaschelli, D., T. Di Matteo, T. Aste, G. Caldarelli and M. Loffredo (2007), "Interplay between topology and dynamics in the World Trade Web", The European Physical Journal B, 57: 1434-6028.

Garlaschelli, D. and M. Loffredo (2004), "Fitness-Dependent Topological Properties of the World Trade Web", Physical Review Letters, 93: 188701.

Garlaschelli, D. and M. Loffredo (2005), "Structure and evolution of the world trade network", Physica A, 355: 138-44.

Haggett, P. and R. Chorley (1969), Network analysis in geography, Edward Arnold, London.

Head, K. and T. Mayer (2010), "Gravity, market potential and economic development", Journal of Economic Geography, : 1-14.

Helliwell, J. F. and T. Padmore (1985), "Empirical Studies of Macroeconomic Interdependence", in R. Jones and P. Kenen, (eds.), Handbook of International Economies, Elsevier Science Publishers B.V.

Helpman, E., M. Melitz and Y. Rubinstein (2008), "Estimating Trade Flows: Trading Partners and Trading Volumes", The Quarterly Journal of Economics, 123: 441-487.

Hillberry, R. and D. Hummels (2005), "Trade Responses to Geographic Frictions: A Decomposition Using Micro-Data", Purdue University, mimeograph.

Hummels, D. (1999), "Towards a geography of trade costs", University of Chicago, mimeograph.

Hummels, D. (2007), "Transportation Costs and International Trade in the Second Era of Globalization", Journal of Economic Perspectives, 21: 131-154.

Jackson, M. O. (2010), Social and Economic Networks, Princeton University Press.

Kali, R., F. Méndez and J. Reyes (2007), "Trade structure and economic growth", Journal of International Trade $\&$ Economic Development, 16: 245-269. 
Kali, R. and J. Reyes (2007), "The architecture of globalization: a network approach to international economic integration", Journal of International Business Studies, 38: 595-620.

Krugman, P. (1995), "Growing World Trade: Causes and Consequences", Brookings Papers on Economic Activity, 26: 327-377.

Krugman, P. R. (1980), "Scale Economies, Product Differentiation, and the Pattern of Trade", American Economic Review, 70: 950-959.

Lawless, M. (2010), "Deconstructing gravity: trade costs and extensive and intensive margins", Canadian Journal of Economics, 43: 1149-1172.

Li, X., Y. Y. Jin and G. Chen (2003), "Complexity and synchronization of the World trade Web", Physica A: Statistical Mechanics and its Applications, 328: 287-96.

McCallum, J. (1995), "National Borders Matter: Canada-U.S. Regional Trade Patterns", American Economic Review, 85: 615-623.

McPherson, M., L. Smith-Lovin and J. M. Cook (2001), "Birds of a Feather: Homophily in Social Networks", Annual Review of Sociology, 27: 415-444.

Melitz, M. J. (2003), "The Impact of Trade on Intra-industry Reallocations and Aggregate Industry Productivity", Econometrica, 71: 1695-1725.

Newman, M. E. J. (2002), "Assortative mixing in networks", Physical Review Letters, 89: $87-101$.

Newman, M. E. J. (2003), "Mixing patterns in networks", Physical Review E, 67: 026126.

Obstfeld, M. and M. Rogoff (2001), "The Six Major Puzzles in International Macroeconomics: Is There a Common Cause?", NBER Macroeconomics Annual 2000, 15: $339-412$.

Redding, S. J. (2011), "Theories of Heterogeneous Firms and Trade", Annual Review of Economics, 3: 77-105.

Redding, S. J. and A. J. Venables (2004), "Economic geography and international inequality", Journal of International Economics, 62: 53-82.

Reichardt, J. and D. White (2007), "Role models for complex networks", The European Physical Journal B, 60: 217-224.

Reyes, J., S. Schiavo and G. Fagiolo (2008), "Assessing the evolution of international economic integration using random-walk betweenness centrality: The cases of East Asia and Latin America", Advances in Complex Systems, 11: 685-702. 
Santos Silva, J. M. C. and S. Tenreyro (2006), "The Log of Gravity", The Review of Economics and Statistics, 88: 641-658.

Serrano, A. and M. Boguñá (2003), "Topology of the World Trade Web", Physical Review E, 68: 015101(R).

Serrano, A., M. Boguñá and A. Vespignani (2007), "Patterns of dominant flows in the world trade web", Journal of Economic and Coordination, 2: 111-124.

Squartini, T., G. Fagiolo and D. Garlaschelli (2011a), "Randomizing World Trade. I: A Binary Network Analysis", Physical Review E, 84: 046117.

Squartini, T., G. Fagiolo and D. Garlaschelli (2011b), "Randomizing World Trade. II: A Weighted Network Analysis", Physical Review E, 84: 046118.

Tinbergen, J. (1962), Shaping the World Economy, The Twentieth Century Fund.

Tzekina, I., K. Danthi and D. Rockmore (2008), "Evolution of community structure in the world trade web", The European Physical Journal B - Condensed Matter, 63: 541-545.

Wei, S. (1996), "Intra-national versus International trade: How Stubborn are Nations in Global Integration?", NBER Working Paper, 5531.

Wilson, A. (2000), Complex Spatial Systems: The Modelling Foundations of Urban and Regional Analysis, Prentice Hall.

WTO (2011), The WTO and preferential trade agreements: From co-existence to coherence. World Trade Report 2011, Geneva: WTO Publications. 